\title{
Wearable Device for Sitting Up Support of Parkinson Patients
}

ISSN: 2576-8816

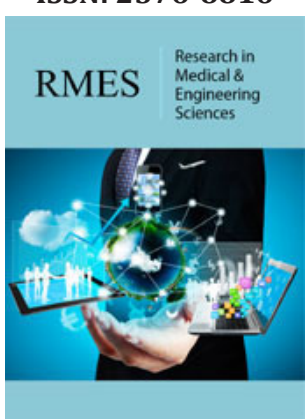

*Corresponding author: Yoshikazu Mori, Department of Intelligent Systems Engineering, Japan

Submission: 侮 June 04, 2019

Published: 惧June 13, 2019

Volume 7 - Issue 5

How to cite this article: Yoshikazu $M$ Shohei K, Keisuke Y. Wearable Device for Sitting Up Support of Parkinson Patients. Res Med Eng Sci. 7(4).RMES.000673.2019. DOI: 10.31031/RMES.2019.07.000673

Copyright@ Yoshikazu Mori, This article is distributed under the terms of the Creative Commons Attribution 4.0 International License, which permits unrestricted use and redistribution provided that the original author and source are credited.

\author{
Yoshikazu Mori ${ }^{1 *}$, Shohei Kojima ${ }^{2}$ and Keisuke Yagi ${ }^{1}$ \\ ${ }^{1}$ Department of Intelligent Systems Engineering, Japan \\ ${ }^{2}$ Seiko Epson Corporation, Japan
}

\begin{abstract}
Purpose: To propose a novel supporting device to help Parkinson patients to turn over and to take a sitting position on a bed.

Methods: Parkinson patients have difficulty with turning motion, which leads to disability in achieving a sitting up position. Nevertheless, they can walk unaided with a caregiver in cases where conditions are not so severe. The device prototype is a vest on which air actuators are attached. We devised air actuators of three kinds comprising piled plastic sheets: fan-type, bellows-type, and split-type. Air actuators push the patient body up as they are expanded by an air compressor.
\end{abstract}

Result: Experiments to verify the prototype device were conducted with healthy participants. Each turning over and sitting up motion was realized automatically using an accelerometer and a digital flow switch.

Conclusion: Results indicate that the performance of the device is sufficient to help the user to complete the sitting up motion on a bed.

\section{Background}

Parkinson disease, a degenerative brain disorder, affects many people worldwide. In Japan in 2011, 116,536 patients had symptoms and brain dysfunction commonly associated with Parkinsonism [1]. Moreover, Parkinson disease is an increasingly common neurological disorder in an aging society. The most common characteristic clinical signs are tremor, muscular rigidity, postural instability, bradykinesia, and hypokinesia caused by a loss of brain dopaminergic neurons [2]. Disabilities of the neck and trunk prevent the upper body from moving freely. Consequently, a person with Parkinson disease has difficulty with turning motion on a bed. Various devices and robots have been developed for nursing care. Some electronic nursing beds have functions for adjustment of the backrest, footrest, and height. A person can rise from a supine position but sitting on the bed and putting the legs down on the floor is difficult to because a person has difficulty with turning motion. Therefore, leaving the bed independently is troublesome for Parkinson patients. Noritsugu developed a mat that can prevent bedsores [3]. A user's body is supported by 24 independently controlled balloon-like units. The maximum body tilt angle is $30 \mathrm{deg}$, which is insufficient to turn over.

\section{Methodology}

Parkinson disease is classified into stages of based on the Hoehn and Yahr Scale [4]. At stage I, patients have unilateral involvement only, usually with minimal or no functional impairment. At stage II, patients have bilateral or midline involvement without impaired balance. At stage III, patients are still physically capable of leading independent lives. Their disability is mild to moderate. At stage IV, patients have severely disabling disease; they can walk and stand unassisted but are markedly incapacitated. At stage V, patients are confined to a bed or wheelchair unless aided. The main targets of the device described herein are patients of stages II-IV [5].

Figure 1 presents the device appearance. It is a vest with attached air actuators. Therefore, its manufacturing cost is low. Moreover, the maximum body tilt angle is greater than in the case in which the actuators are attached to a bed. The user need not change the bed. The vest can be put on and taken off easily because one side of it can be opened. We devised air 
actuators of three kinds as depicted in Figure 2: fan type, bellows type, and split type. The air actuators push the patient body up as they are expanded by an air compressor. The air actuator material is a multilayer film of nylon and polyethylene. The fantype actuator expands similarly to a fan. Turning over a body in the transition from a face-up position to a lateral position is effective. The bellows-type actuator is used for magnifying the amount of displacement. The split-type actuator is useful for increasing upperbody stability when sitting up. One fan-type and one bellows-type $(\mathrm{W}=20 \mathrm{~cm}, \mathrm{H}=25 \mathrm{~cm})$ air actuator are attached to the upper right position of the vest for turning over, whereas one split-type and two bellows-type $(\mathrm{W}=30 \mathrm{~cm}, \mathrm{H}=21 \mathrm{~cm})$ actuators are attached to the lower left position of it for sitting up (Figure 1). Each air actuator swells one by one. The tilt angle of the body is measured using a triaxial accelerometer (KXR94-2050; Kionix Inc.). We used an air pump (TZX512/V-12; Top Shelf Pumps) to supply air to the device. A digital flow switch (PFM725-C6-C-M; SMC Corp.) opens and closes the air valve according to the air amount. A microcomputer (Arduino Uno) controls the system.

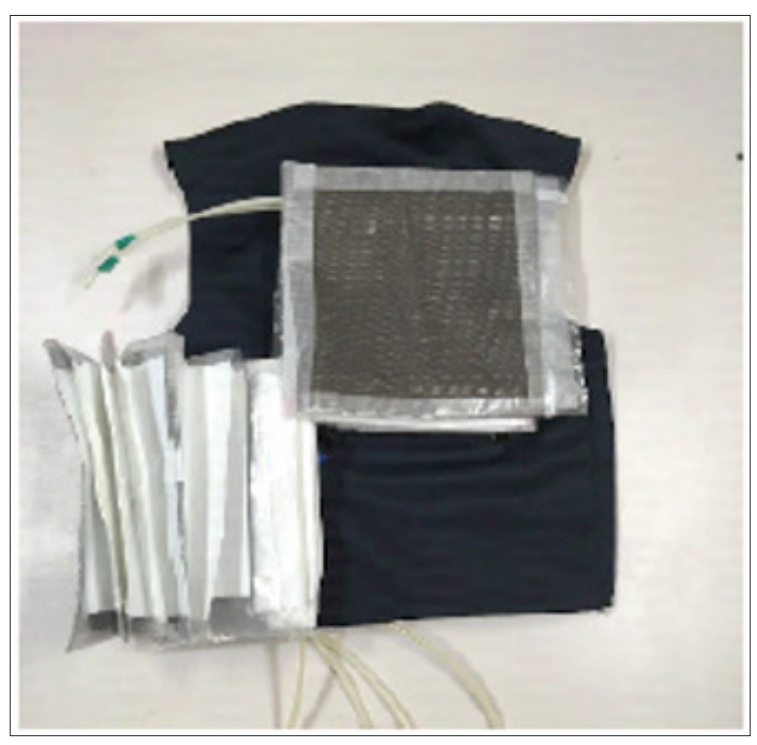

Figure 1: Proposed device.

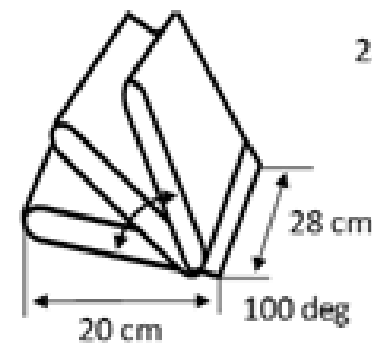

(a) Fan type

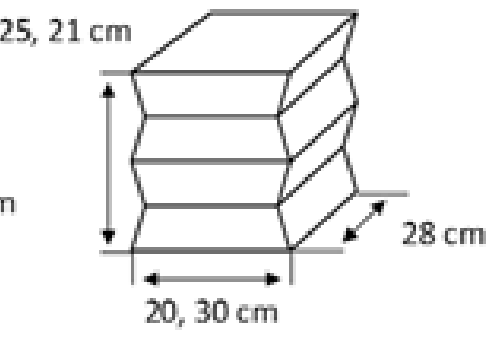

(b) Bellows type

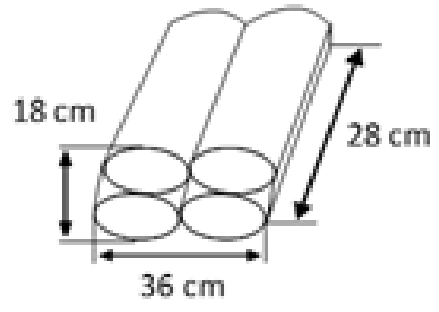

(c) Split type

Figure 2: Three types of the air actuator.

\section{Results}

Figure 3 presents photographs of an experiment on a bed. The subject was a healthy man of $170 \mathrm{~cm}$ height with no Parkinson disease. His weight was $54 \mathrm{~kg}$. He consented to participate in the study after the purpose, procedures, and necessity of this study were explained, and after he was promised anonymity and protection of personal information. The sequence of motion is the following, as portrayed in the panels of Figure 3: (a)-(b) The subject took a face- up supine position with his arms crossed. He bent his knees. A start button was pressed to send the start signal to the microcomputer. The air actuators attached on the upper right position of the vest pushed the upper body. As a result, the subject turned over. (b)(d) The air actuators attached on the lower left position pushed the user up gradually. Then he sat up on the bed. (e)-(f) The subject took the vest off and left the bed. (g)-(h) The subject put on the vest again and lay down on the bed.
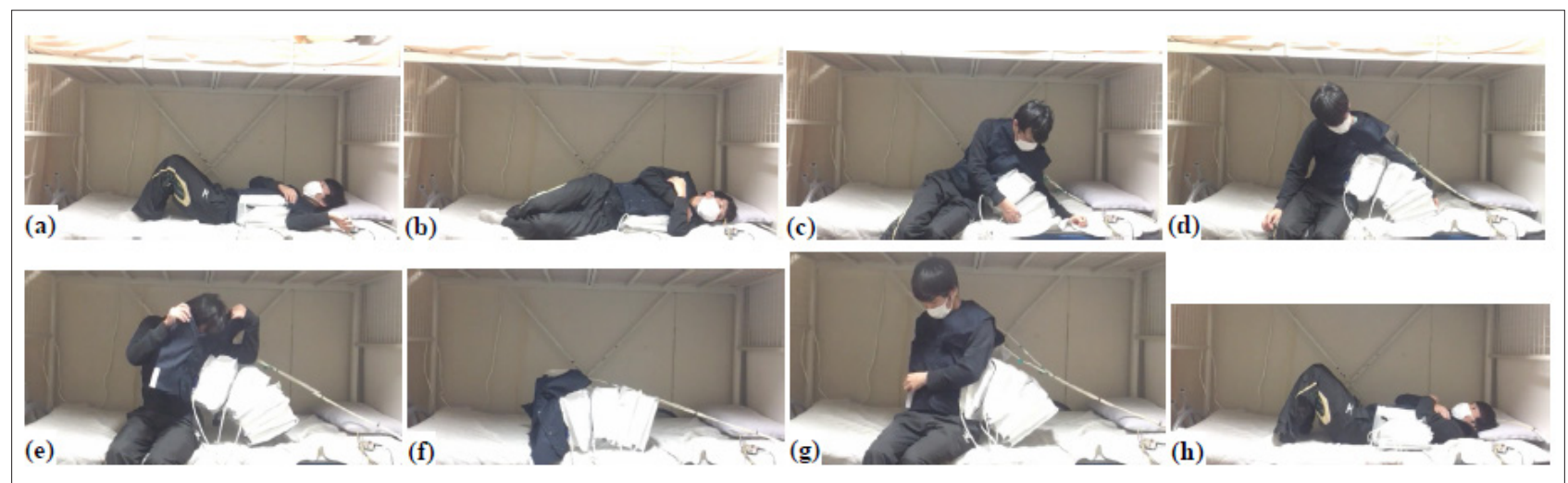

Figure 3: Sitting up (a)-(d) and falling (g)-(h) on a bed. 


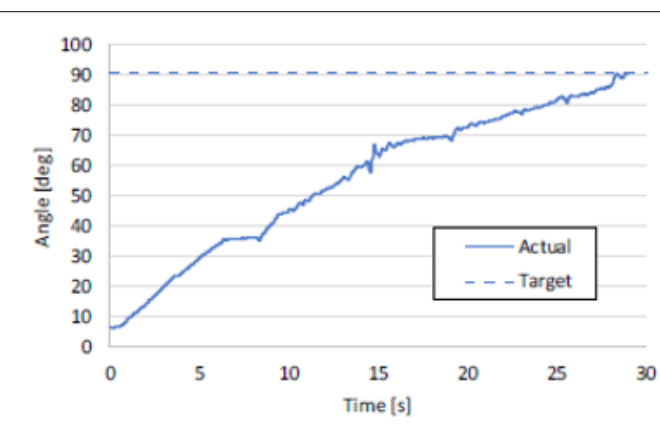

(a) Roll angle of the upper body when turning over.

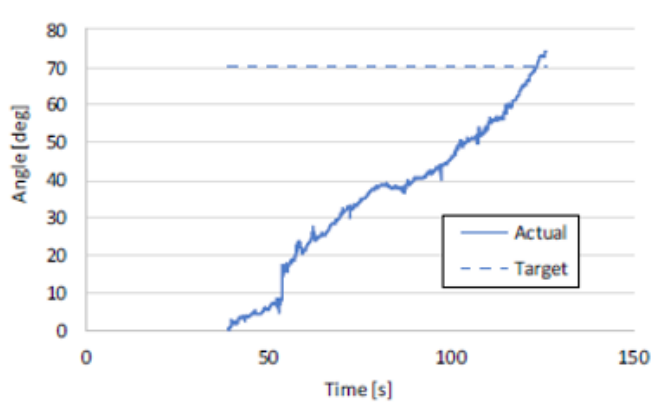

(b) Pitch angle of the upper body when sitting up.

Figure 4: Experimental results.

Figure 4 presents results obtained when turning over and sitting up. Each turning over and sitting up motion was realized automatically using an accelerometer and a digital flow switch. The respective target angles when turning over and sitting up were $90 \mathrm{deg}$ and $70 \mathrm{deg}$. The latter was set based on the posture when the subject sat on the bed supporting the upper body with a stretched arm. Each motion was conducted smoothly. Five subjects with no Parkinson disease (male, average height of $169.2 \pm 2.48 \mathrm{~cm}$ ) were able to sit up on the bed similarly.

\section{Conclusion}

We proposed a supporting device for Parkinson patients to sit up on a bed. Experimentation demonstrated its effectiveness. In future studies, this device can be improved for better practical use and can be tested for Parkinson patients under secure conditions.

\section{References}

1. Japan Intractable Diseases Information Center (2019) The number of medical recipient certificate holder for specific disease treatment, Japan.

2. Butt AH, Rovini E, Dolciotti C, Bongioanni P, Petris GD, et al. (2017) Leap motion evaluation for assessment of upper limb motor skills in parkinson's disease. International Conference on Rehabilitation Robotics, London, UK, pp. 17-20.

3. Noritsugu T, Igata T, Takaiwa M and Sasaki D (2005) Development of Medical Care Mat for Prevention of Bedsore. Journal of the Japan Society of Mechanical Engineers 71(703): 187-194 (in Japanese).

4. Hoehn M and Yahr M (1967) Parkinsonism: onset, progression and mortality. Neurology 17: 427-442.

5. Kojima S, Mori Y and Yagi K (2019) Device Supporting Sitting Up Motion for Parkinson's Patients. Journal of Life Support Engineering, Japan. 